\title{
Differences between Scheimpflug and optical coherence tomography in determining safety distances in eyes with an iris-fixating phakic intraocular lens
}

\author{
Zoraida S. Gaurisankar $^{1,2}$ (D) Gwyneth A. van Rijn ${ }^{1}$ (D) Gregorius P. M. Luyten ${ }^{1}$ (D) Jan-Willem M. Beenakker ${ }^{1,3}$ (D)
}

Received: 14 May 2020 / Revised: 17 July 2020 / Accepted: 27 July 2020 / Published online: 6 August 2020

(C) The Author(s) 2020

\begin{abstract}
Purpose To investigate the agreement and reliability of anterior segment optical coherence tomography (AS-OCT) and Scheimpflug imaging in measuring the distance from the anterior edge of an iris-fixated phakic intraocular lens (IF-pIOL) to the corneal endothelium.

Methods Anterior segment configuration was assessed in a total of 62 eyes of which 25 hyperopic and 37 myopic eyes, all corrected with an IF-pIOL. Measurements were performed by two independent observers using AS-OCT (Visante, Model 1000, Carl Zeiss Meditec Inc.) and Scheimpflug imaging (Pentacam HR, Oculus Optikgerate). The distance from the anterior edge of the pIOL to the endothelium was measured in five different positions using both modalities with their corresponding pIOL software. The measurements as well as the inter- and intra-observer reliability of the two imaging modalities were then compared.

Results Distance measurements for all positions performed by AS-OCT were found to be significantly larger than those performed by Scheimpflug imaging, with mean differences ranging from 0.11 to $0.22 \mathrm{~mm}$. Both instruments exhibited good interand intra-observer reliability.

Conclusion Anterior pIOL edge to endothelium distance measurements by AS-OCT and Scheimpflug imaging have good intraand inter-observer reliability. However, as AS-OCT provides larger measurements, these two modalities cannot be used interchangeably. Correction of this difference might be essential for proper decision-making during pre-operative screening for pIOL implantation and post-operative safety monitoring.
\end{abstract}

Keywords Phakic intraocular lens $\cdot$ Lens position $\cdot$ PENTACAM $\cdot$ Scheimpflug $\cdot$ Visante OCT $\cdot$ Anterior segment OCT

Zoraida S. Gaurisankar and Gwyneth A. van Rijn are co-first authors.

Electronic supplementary material The online version of this article (https://doi.org/10.1007/s00417-020-04874-7) contains supplementary material, which is available to authorized users.

\section{Zoraida S. Gaurisankar}

1 Department of Ophthalmology, Leiden University Medical Center, Leiden, the Netherlands

2 Leiden, the Netherlands

3 Department of Radiology, C.J. Gorter Center for High-Field MRI, Leiden University Medical Center, Leiden, the Netherlands

\section{Introduction}

Phakic intraocular lens (pIOL) implantation has proven to be safe and effective for the correction of a broad range of ametropia [1, 2]. The Artisan lens (Ophtec BV, Groningen, the Netherlands) is an iris-fixated (IF) pIOL that has been used successfully to correct moderate to high myopia, hyperopia, and astigmatism since 1991. The outcomes after Artisan implantation have found to be predictive and stable over time [1, $3,4]$.

To establish the long-term safety of IF-pIOL and to prevent complications, an extensive pre-operative evaluation in combination with long-term post-operative follow-up is required. One of the most feared and important potential complications of any type of anterior segment surgery is accelerated 
endothelial cell (EC) loss, especially in the case of IF-pIOL. As this risk has been shown to be negatively correlated to the anterior chamber depth, the position of an IF-pIOL in the anterior chamber is one of the main safety parameters in both pre-operative screening and follow-up [1, 4-9].

Monitoring of the anatomical relationship with an IF-pIOL in the eye can be performed at the slit lamp. However, the accuracy between the distance of the pIOL to the corneal endothelium is subject to subjective interpretation and is thus limited. To objectively measure the distance between the central and peripheral pIOL edge to the corneal endothelium, several clinical techniques may be used, including ultrasound biomicroscopy (UBM), Scheimpflug imaging, and anterior segment optical coherence tomography (AS-OCT). UBM delivers images of excellent quality but has several limitations, such as the fact that it is technically challenging, with a risk of distorting the true anterior chamber dimension, timeconsuming to perform, and possibly uncomfortable for the patients [10]. The non-contact AS-OCT [11-13] and Scheimpflug imaging techniques [14-16] both provide highresolution images of the anterior chamber on which the pIOL position can be determined with the provided software.

To minimize the risk of increased cell loss, Baikoff introduced in 2006 the "minimum (or "critical') safety distance": a minimum distance between the central edge of the optical zone of the pIOL and the endothelium [11]. Based on the clinical results of PérezSantonja et al. [17] and de Sousa et al. [18], he proposed a minimum distance of $1.5 \mathrm{~mm}$ to prevent accelerated EC loss. Later studies confirmed the importance of the central distance between the anterior surface of the $\mathrm{pIOL}$ and the endothelium $[13,15,16,19]$, showing a yearly increase in EC loss with smaller distances. Doors et al. described an average EC loss of $0.15 \%, 0.98 \%$, and $1.80 \%$ per year for a minimum central distance between the anterior surface of the pIOL and the endothelium of $1.59 \mathrm{~mm}, 1.37 \mathrm{~mm}$, and $1.15 \mathrm{~mm}$, respectively [13]. In addition to the central distances and a smaller ACD, Jonker et al. [19] found smaller distances between the peripheral pIOL edge and endothelium to also be a significant risk factor for accelerated EC loss.

The aim of this study is to compare the AS-OCT and Scheimpflug imaging in measuring pIOL-to-endothelium distances and to assess the inter- and intra-observer variability of these measurements.

\section{Methods}

In this cross-sectional study, we examined 62 phakic eyes that had undergone pIOL implantation, of which 25 eyes (13 patients) were corrected for hyperopia and 37 eyes (20 patients) for myopia. All the eyes were implanted with an Artisan IF-pIOL by the same experienced eye surgeon at the Leiden University Medical Center (LUMC), Leiden, or Erasmus Medical Center,
Rotterdam; Artisan lens model 203 was implanted for hyperopia and model 206 for myopia, with the available refractive powers ranging from +1.0 to +12.0 diopters and -1.0 to -23.5 diopters respectively, in 0.5 diopter steps. The study was approved by the Medical Ethical Committee of the LUMC and was conducted in accordance with the Declaration of Helsinki. Informed consent was obtained from all patients before they were examined. Anterior segment scans were made with two different imaging modalities: the AS-OCT and Scheimpflug imaging. All images were made under the same dim light conditions in an unaccommodated state.

The Visante OCT (Visante, Model 1000, software version 3.0.1.8, Carl Zeiss Meditec Inc.) is a time-domain system that uses infrared light $(1310 \mathrm{~nm})$ to image the anterior segment. For this study, all measurements were performed in highresolution mode, which provides a detailed image with a field of view of $10 \mathrm{~mm}$ width by $3 \mathrm{~mm}$. In this mode, the Visante performs 512 scans to assess the anterior segment area in 0.25 s. Axial and transverse resolutions are 18 and $60 \mu \mathrm{m}$, respectively.

The Pentacam HR system (Pentacam HR, software version 1.12r24, Oculus Optikgerate) uses the Scheimpflug imaging technique for anterior segment evaluation. A $360^{\circ}$, rotating, non-contact camera uses a monochromatic slit light source to reconstruct a three-dimensional map of the anterior segment of the eye. Such a scan is performed in $2 \mathrm{~s}$ and yields images with a clear visualization of the pIOL. For assessing the pIOL position, a 3-D pIOL-simulation software module is provided.

The acquired images were subsequently analyzed using the vendors' software. With AS-OCT, the distance from the pIOL to the corneal endothelium is measured by manually placing a pIOL template on the anterior segment image by computer mouse selection and dragging and drawing a measurement vector using the vendor's software (Fig. 1a, b). In the case of Scheimpflug imaging, the software automatically calculates the minimum distance between the pIOL and the corneal endothelium after the 3-D pIOL template is manually added to the image (Fig. 1c, d). When present, the iris image is used for better precision of the pIOL template position. On both types of anterior segment scans, the pIOL-to-endothelium distance was measured in five standard positions along the $180^{\circ}$ horizontal axis (at "3 o'clock" and "9 o'clock" positions) (Fig. $1 b, d)$ :

- Central

- At $2.5 \mathrm{~mm}$ nasal from the center

- At $2.5 \mathrm{~mm}$ temporal from the center

- At $4 \mathrm{~mm}$ nasal from the center

- At $4 \mathrm{~mm}$ temporal from the center

To determine the inter- and intra-observer variability, these analyses were performed separately by two independent, 


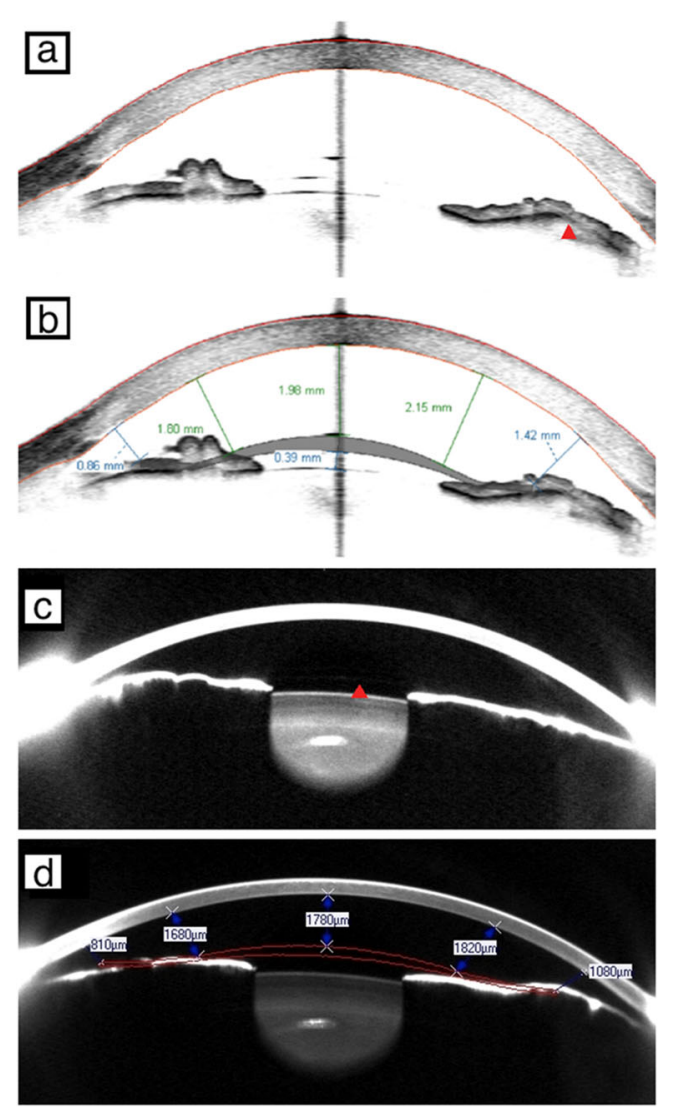

Fig. 1 Anterior segment scan image acquired with the Visante anterior segment optical coherence tomography (AS-OCT) before (a; red arrow: phakic intraocular lens (pIOL) enclavation site) and after placement of the pIOL template using the pIOL analysis software (b). Similar images acquired with Scheimpflug imaging before (c; red arrow: edge of pIOL) and after placement of pIOL template (d; contrast of scan was adjusted). All four scans represent the left eye of the same subject on a $180^{\circ}-0^{\circ}$ axis. (Please note the differences in clearance distances given by the Pentacam compared with the Visante)

trained observers (ZSG, GAR). Both observers repeated the measurements at another time point, at least 3 months from the first measurements and without knowledge of the earlier results. To test the agreement between the two imaging modalities, the average of all four measurements was used for analysis.

\section{Statistical analysis}

All statistical analyses were performed using SPSS statistics software version 25 (SPSS Inc., IBM, Somers, NY).

To assess the agreement between tomographers, a paired sample $t$ test was applied and the Bland-Altman analysis was performed, and 95\% limits of agreement (LoA) were estimated by the mean difference $\pm 1.96 \times$ standard deviation $(\mathrm{SD})$ of the difference [20]. To exclude potential cofounding factors (right or left eye, hyperopic or myopic eye, time interval between pIOL implantation and examination date), a linear mixed model was used where these factors were taken into account to test their significance. A $p$ value of $<0.05$ was considered to be statistically significant.

Inter- and intra-observer reliability was assessed by calculating the intra-class correlation coefficients (ICC) using a multilevel (hierarchical) linear mixed model to adjust for the possible correlation between measurements within the same eye and between the two eyes within the same patient. In this model, intra-observer reliability was evaluated by correlating each observer's first measurement by AS-OCT and Scheimpflug imaging with the same observer's second measurement. Inter-observer reliability was assessed by correlating measurements of one observer with the corresponding measurements of the other observer. The ICC was interpreted according to Cohen's kappa classification [21].

\section{Results}

\section{Patient characteristics}

Sixty-two phakic eyes of 34 subjects including 11 males and 23 females between the age of 24.9 and 76.6 years, with a mean (SD) of 49.6 (11.2) years, were examined. The power of the Artisan lenses implanted ranged from +12.00 to 23.50 diopters. The mean time interval between pIOL implantation and the first anterior segment analysis was 9.7 (4.7) years. For more details, see Table 1.

\section{Inter- and intra-observer reliability}

The overall inter-observer ICC was 0.99 with a $95 \%$ confidence interval (CI) of 0.99-0.99 for both AS-OCT and Scheimpflug imaging. The overall intra-observer ICC was 0.99 with a $95 \%$ CI of $0.99-0.99$ for AS-OCT and 0.98 with a $95 \%$ CI of $0.98-0.98$ for Scheimpflug imaging. The ICCs per position measurement of each instrument are shown in Table 2. All correlations were "very good" for both ASOCT and Scheimpflug imaging according to Cohen's kappa classification [21], showing that a single measurement is reliable irrespective of observer or measurement occasion.

\section{Agreement between instruments}

The distance from the anterior edge of the pIOL to the endothelium when measured by AS-OCT was consistently larger than when measured by Scheimpflug imaging, for all five separate positions, as listed in Table 3. The mean difference for all of the various positions was $0.161(0.120) \mathrm{mm}$ with a 95\% LoA of -0.074 and 0.396 (paired $t_{309}=23.74 ; p<$ 0.001), see Fig. 2 for the Bland Altman plot. The peripheral measurements showed similar results. Supplementary Fig. 1 shows the Bland-Altman plots for the differences in distance measurements at the 5 positions with the $95 \%$ LoA and $95 \%$ 
Table 1 Patient characteristics

\begin{tabular}{lccc}
\hline Variable & Total & Hyperopic eyes & Myopic eyes \\
\hline Eyes (count) & 62 & $25(12$ right eyes) & $37(17$ right eyes $)$ \\
Sex (male:female) [\%] & $32: 68$ & $64: 36$ & $11: 89$ \\
Age at examination \pm SD (min-max) [years] & $49.6 \pm 11.2(24.9-76.6)$ & $52.6 \pm 9.3(24.9-67.4)$ & $47.6 \pm 12.0(25.9-76.6)$ \\
pIOL power \pm SD (min-max) [D] & $9.7 \pm 4.7(0.0-18.0)$ & $7.7 \pm 2.6(2.0-12.0)$ & $-13.6 \pm 4.6(-23.5$ to -13.6$)$ \\
Time interval between pIOL implantation & & $9.8 \pm 3.6(0.0-14.0)$ & $9.5 \pm 5.5(0.0-18.0)$ \\
$\quad$ and anterior segment examination \pm SD & & & \\
$\quad$ (min-max) [years] & & & \\
\hline
\end{tabular}

$S D$ standard deviation; $p I O L$ phakic intraocular lens; $D$ diopters

CIs. The mean difference between AS-OCT and Scheimpflug imaging for the central distance measurements was $0.150 \mathrm{~mm}$ (95\% LoA, -0.014 and 0.314 ), for $2.5 \mathrm{~mm}$ nasal $0.189 \mathrm{~mm}$ (95\% LoA, -0.020 and 0.398 ), for $2.5 \mathrm{~mm}$ temporal $0.114 \mathrm{~mm}$ (95\% LoA, -0.102 and 0.330 ), for $4.0 \mathrm{~mm}$ nasal $0.218 \mathrm{~mm}(95 \% \mathrm{LoA},-0.045$ and 0.481$)$, and for $4.0 \mathrm{~mm}$ temporal $0.137 \mathrm{~mm}$ (95\% LoA, -0.115 and 0.389$)$. In a mixed model, distance measurements were not found to be significantly affected by age, sex, right or left eye, hyperopic or myopic eye, or the time interval between pIOL implantation and the examination date, so these factors were not included in further analyses.

Subsequently, a generalized estimating equation (GEE) model was developed. In this model, we used the average of four repeated analyses (each analysis was acquired twice by both the first and the second observer) of the different distances with the average AS-OCT measurements as the dependent variable and the average Scheimpflug measurements as the independent variable. To assess the effect of the position of the measurement on this comparison, the same model was repeated with "position" as the fixed factor. Following this model, the measurements of the two devices were correlated with the standardized regression coefficient $(r)$ of $0.962(p<$ 0.001 ), with larger distances being measured by AS-OCT than by Scheimpflug imaging. Linear regression analysis yielded the following correlation (Eq. 1: correlation of AS-OCT and Scheimpflug for pIOL-to-endothelium distance measurements):

$D_{\mathrm{AS}-\mathrm{OCT}}=0.962 \times D_{\text {Scheimpflug }}+0.212 \mathrm{~mm}$

where $D$ is the pIOL-to-endothelium distance (in millimeters)

This relation is clearly visible in the scatter plot of Fig. 3 . To assess if this "overall" regression coefficient accounts for all distance positions separately, each regression coefficient of a position was compared with the average regression coefficient of the other positions using linear regression. For every clearance distance position, the regression coefficient did not significantly differ from the others, indicating that there was no effect of the different "distance position" slopes.

\section{Discussion}

Correct positioning of an IF-pIOL in the anterior chamber is of high importance to determine long-term safety, as a smaller $\mathrm{ACD}$ and smaller distance from the edge of the pIOL to the

Table 2 Intra-class correlation coefficients of anterior segment optical coherence tomography and Scheimpflug imaging show good reproducibility of analysis for both modalities

\begin{tabular}{|c|c|c|c|c|}
\hline & \multicolumn{2}{|l|}{ AS-OCT } & \multicolumn{2}{|l|}{ Scheimpflug imaging } \\
\hline & \multicolumn{2}{|l|}{ ICC } & \multicolumn{2}{|l|}{$\mathrm{ICC}$} \\
\hline & Inter-observer $(95 \% \mathrm{CI})$ & Intra-observer $(95 \% \mathrm{CI})$ & Inter-observer $(95 \% \mathrm{CI})$ & Intra-observer $(95 \% \mathrm{CI})$ \\
\hline $4.0 \mathrm{~mm}$ nasal endothelium to $\mathrm{pIOL}$ & $0.944(0.908-0.966)$ & $0.917(0.882-0.942)$ & $0.890(0.813-0.935)$ & $0.818(0.740-0.873)$ \\
\hline $2.5 \mathrm{~mm}$ nasal endothelium to $\mathrm{pIOL}$ & $0.969(0.949-0.982)$ & $0.961(0.944-0.972)$ & $0.958(0.928-0.976)$ & $0.913(0.875-0.939)$ \\
\hline central endothelium to $\mathrm{pIOL}$ & $0.996(0.994-0.998)$ & $0.909(0.835-0.949)$ & $0.955(0.910-0.976)$ & $0.991(0.987-0.994)$ \\
\hline $2.5 \mathrm{~mm}$ temporal endothelium to $\mathrm{pIOL}$ & $0.946(0.911-0.968)$ & $0.930(0.901-0.951)$ & $0.965(0.940-0.979)$ & $0.944(0.920-0.961)$ \\
\hline $4.0 \mathrm{~mm}$ temporal endothelium to $\mathrm{pIOL}$ & $0.955(0.910-0.976)$ & $0.948(0.926-0.964)$ & $0.955(0.920-0.974)$ & $0.919(0.884-0.944)$ \\
\hline
\end{tabular}

$A S-O C T$ anterior segment optical coherence tomography; ICC intra-class correlation coefficient; $95 \% C I$ 95\% confidence interval; $p I O L$ phakic intraocular lens 
Table 3 Means and differences in distance measurements made by anterior segment optical coherence tomography and Scheimpflug imaging

\begin{tabular}{lllllll}
\hline & AS-OCT & Scheimpflug & \multicolumn{2}{l}{ AS-OCT versus Scheimpflug } \\
\cline { 5 - 8 } Measurement to endothelium [mm] from & Mean \pm SD & Mean \pm SD & Difference (mean \pm SD) & Range & $95 \%$ CI & $p$ value \\
\hline 4.0 mm nasal of anterior edge of pIOL & $1.018 \pm 0.249$ & $0.799 \pm 0.231$ & $0.218 \pm 0.135$ & $0.30-1.52$ & $0.184-0.253$ & $<0.001$ \\
$2.5 \mathrm{~mm}$ nasal of anterior edge of pIOL & $1.652 \pm 0.282$ & $1.462 \pm 0.251$ & $0.189 \pm 0.107$ & $0.90-2.31$ & $0.162-0.217$ & $<0.001$ \\
center of anterior edge of pIOL & $2.184 \pm 0.361$ & $2.034 \pm 0.362$ & $0.150 \pm 0.084$ & $1.17-2.78$ & $0.129-0.171$ & $<0.001$ \\
$2.5 \mathrm{~mm}$ temporal of anterior edge of pIOL & $1.760 \pm 0.271$ & $1.647 \pm 0.261$ & $0.113 \pm 0.111$ & $1.11-2.40$ & $0.085-0.142$ & $<0.001$ \\
4.0 mm temporal of anterior edge of pIOL & $1.180 \pm 0.280$ & $1.043 \pm 0.263$ & $0.137 \pm 0.128$ & $0.46-1.89$ & $0.104-0.170$ & $<0.001$ \\
All five positions of anterior edge of pIOL & $1.509 \pm 0.509$ & $1.397 \pm 0.517$ & $0.161 \pm 0.120$ & $0.30-2.78$ & $0.148-0.175$ & $<0.001$ \\
\hline
\end{tabular}

$A S-O C T$ anterior segment optical coherence tomography; 95\% CI 95\% confidence interval; pIOL phakic intraocular lens

endothelium can cause accelerated EC loss, which could lead to the need for early pIOL removal [19, 22]. Jonker et al. have recently reported a prevalence of IF-pIOL explantation due to excessive EC loss of up to $6.0 \%$ during 5- and 10-year followup [19]. Today, both AS-OCT and the Scheimpflug imaging are used to measure the pIOL edge to endothelium distance before and after pIOL implantation [11, 13, 15, 18]. The overall reproducibility of ACD biometry before and after pIOL implantation has been documented for both imaging modalities [23, 24], and a comparison study for ACD has shown a significant difference between the AS-OCT and Scheimpflug [23]. However, no reproducibility or comparison studies of the pIOL edge to endothelium distance measured with these two different imaging modalities have been performed. In this study, we demonstrate good inter- and intra-observer reproducibility for AS-OCT and Scheimpflug imaging when performing these measurements. A comparison between the two modalities, however, shows a significant difference in the measurement of the pIOL edge to endothelium distance, with the AS-OCT measurements being consistently larger than the Scheimpflug measurements.

Let us take a brief look at the aspects that differ between these instruments: firstly, the Pentacam HR, which uses Scheimpflug imaging, provides good images of the anterior segment. However, complex geometrical adjustments are performed to correct optical distortions caused by this modality $[25,26]$. With AS-OCT, these optical corrections do not need to be made for axial measurements. However, for peripheral

Fig. 2 Bland-Altman plot showing the difference in distance measurements between the anterior segment optical coherence tomography and Scheimpflug imaging modalities for all positions from the anterior phakic intraocular lens (pIOL) to the endothelium. The red line represents the mean, the black line the upper and lower 95\% confidence interval, the dashed lines the upper and lower 95\% limits of agreement (LoA). Triangles: hyperopic eyes; dots: myopic eyes

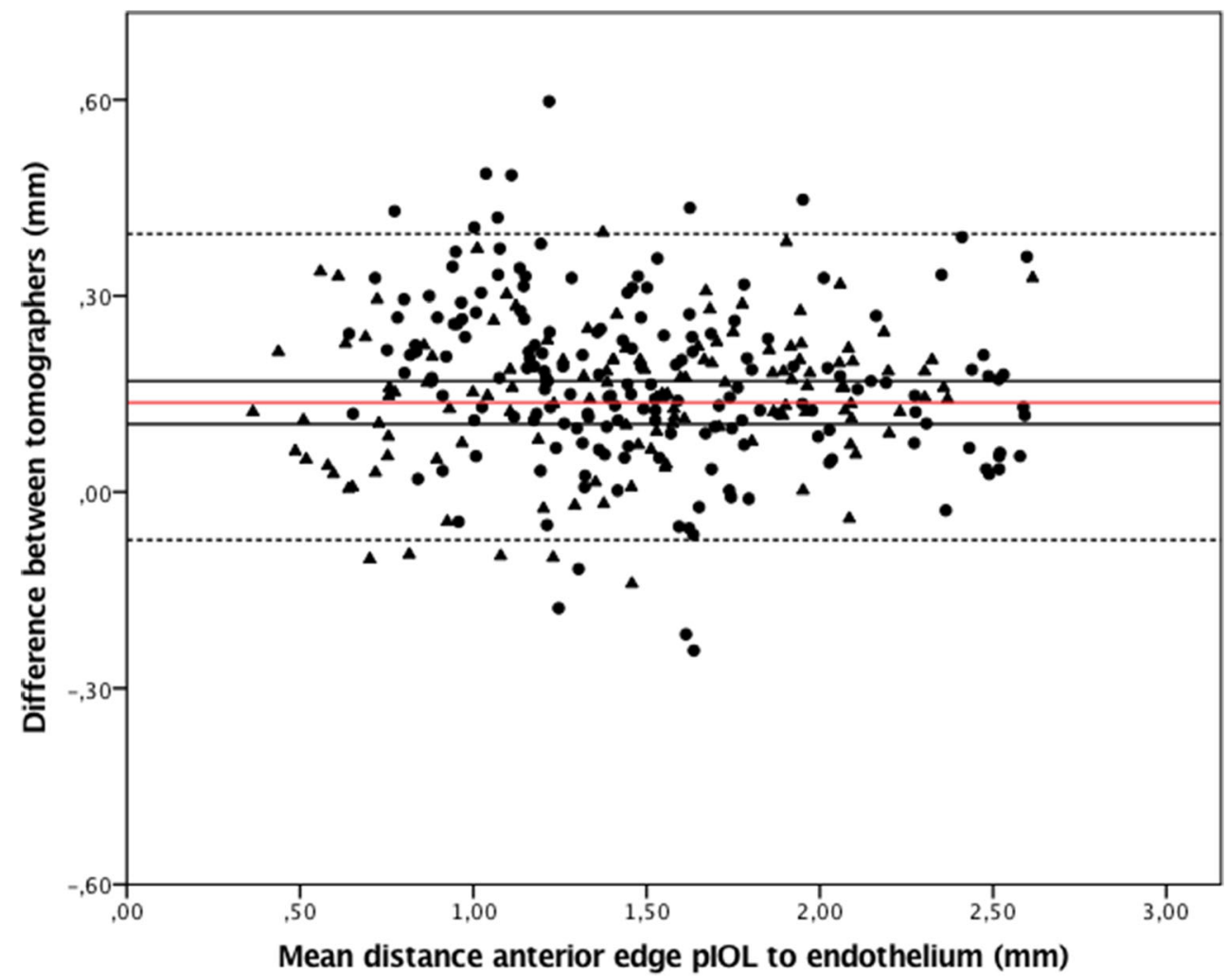


Fig. 3 Scatter plot of the anterior segment optical coherence tomography (AS-OCT) measurements against Scheimpflug imaging measurements. The regression fit line (black line) following the relationship of the devices consistently shows higher measurements of AS-OCT compared with the dashed line which represents the absolute agreement of the instruments. Dot colors represent the positions of distances from the pIOL to the endothelium: red: central; green: $2.5 \mathrm{~mm}$ temporal from the center; orange: $2.5 \mathrm{~mm}$ nasal from the center; yellow: $4.0 \mathrm{~mm}$ nasal from the center; blue: $4.0 \mathrm{~mm}$ nasal from the center

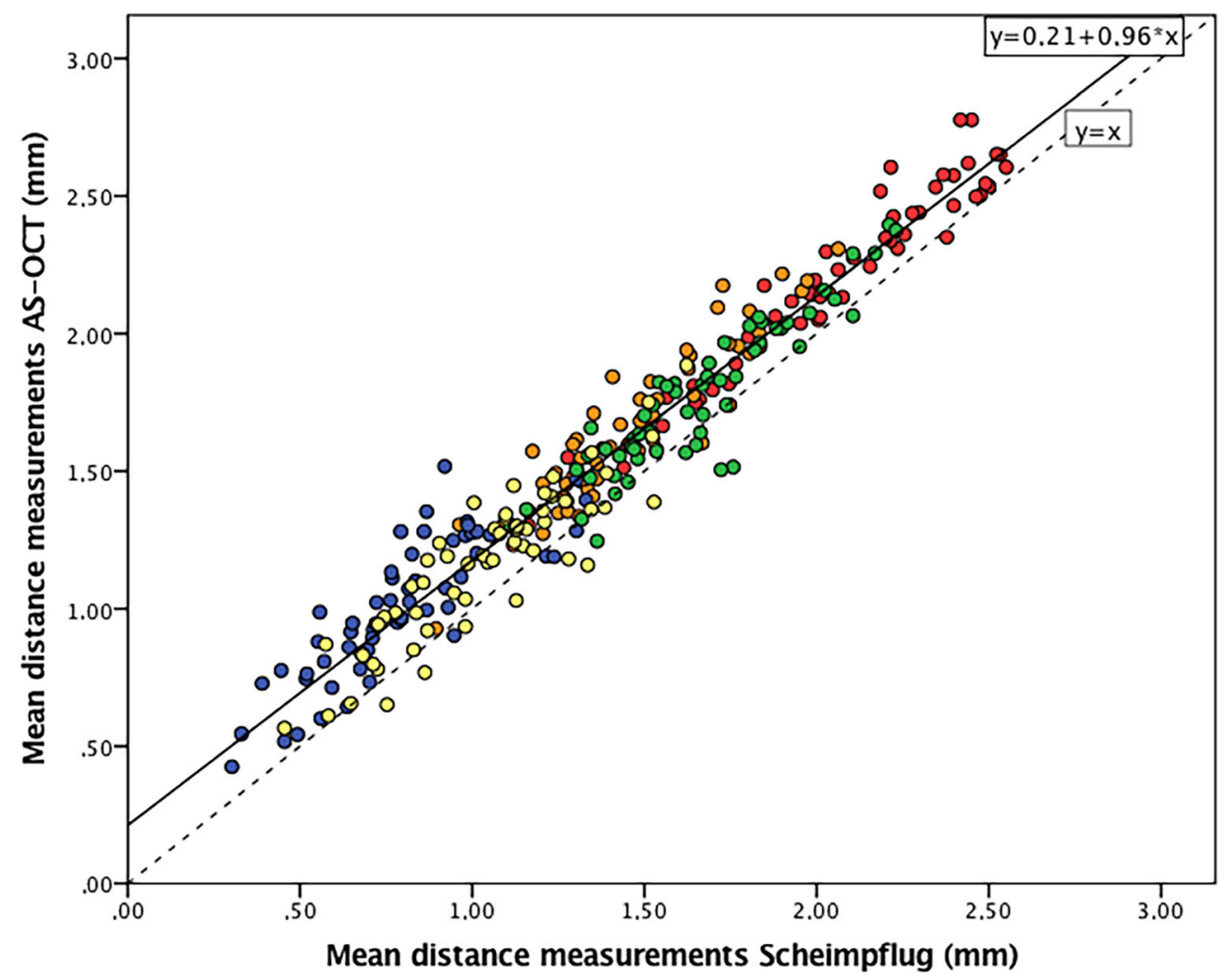

measurements, refraction at the corneal surface will result in a systematic error [27]. Moreover, based on this study, similar differences between OCT techniques, such as spectrometerbased and swept-source OCT, are plausible as these use different optical setups [28] which might result in similar systematic differences in apparent pIOL-to-endothelium distances. Secondly, we need to consider the effect of the different software instructions to measure the pIOL-to-endothelium distance: With the Pentacam software, minimum pIOL-toendothelium distances are automatically identified and visualized for different positions after aligning the 3-D pIOL template. By contrast, the OCT calculations are based on manually defined distances since both the pIOL template and all the different distances are manually dragged and drawn (vector tool) onto the 2-D anterior segment scan. Although this manual interaction could reduce the inter- and intraobserver reproducibility, especially for less trained operators, it cannot explain the systematic difference between both devices.

Different models and minimum ("critical") pIOL-toendothelium distances are described in the literature for monitoring anterior chamber pIOL safety. Baikoff [11] at first suggested a minimum safety distance between the pIOL and corneal endothelium of $1.5 \mathrm{~mm}$, a distance based on Scheimpflug results from earlier studies [11, 17]. Doors et al. $[12,13]$ evaluated pIOL clearances with the Visante OCT. Ferreira et al. [22] provided the clinicians with a new safety reference in 2014: a minimum central clearance distance of $1.7 \mathrm{~mm}$, based on their Pentacam results. Recently, Jonker et al. [19] have demonstrated a $10.3 \%$ EC loss over 5 years and $20.5 \%$ over 10 years with a mean distance between the central pIOL edge and endothelium of $2.17 \mathrm{~mm}$ using the Visante AS-OCT. This risk showed a linear increase in EC loss with smaller distances.

For correct interpretation of the previously mentioned "critical minimum pIOL-to-endothelium distance," including the risk of EC loss, the imaging modality used to obtain the pIOLto-endothelium distance should be taken into account, as, according to our results, AS-OCT overestimates this distance compared with Scheimpflug. When using a Scheimpflugbased minimum safety distance for an AS-OCT scan, we suggest the use of our conversion equation. For example, based on Eq. 1, the minimum safety distance should be $1.84 \mathrm{~mm}$, instead of $1.7 \mathrm{~mm}$ as proposed by Ferreira [22], when using AS-OCT. This difference of $0.14 \mathrm{~mm}$ is relevant for the follow-up of the patients, as it could explain increased EC loss. It is, however, important to realize that the found relation between both devices, and therefore also the modified safety distance, is not only vendor but also potentially software version-dependent.

In conclusion, AS-OCT and Scheimpflug imaging measuring the distance from the anterior edge of a pIOL to the corneal endothelium are both accurate with good reproducibility, but the AS-OCT provides consistently larger measurements compared with Scheimpflug imaging. This difference is of great clinical importance for the follow-up of pIOL positioning in 
the anterior chamber. We therefore suggest not to use these two imaging modalities interchangeably for measuring the pIOL-to-endothelium distance during follow-up. Clinicians using a fixed minimum safety distance or predictive model for safety follow-up should be aware of the instrument used for measurement as conversion might be needed.

Acknowledgments The authors thank Janette Symons for assistance with medical writing.

Code availability The data code (SPSS Syntax) that support the findings of this study are available from the corresponding author, ZSG, upon reasonable request.

Author contributions All authors contributed to the study conception and design. Material preparation, data collection, and analysis were performed by ZS Gaurisankar, GA van Rijn, and GPM Luyten. The first draft of the manuscript was written by ZS Gaurisankar and all authors commented on previous versions of the manuscript. All authors read and approved the final manuscript.

Funding information The authors were supported by Opthec b.v., Stichting Blindenhulp, and by the following foundations through UitZicht: ANVVB and LSBS.

Data availability The data that support the findings of this study are available from the corresponding author, $\mathrm{ZSG}$, upon reasonable request.

\section{Compliance with ethical standards}

Conflict of interest G.A. van Rijn received research grants from Ophtec b.v. All other authors declare that they have no conflict of interest.

Ethics approval Approval was obtained from the ethics committee of Leiden University Medical Center. The procedures used in this study adhere to the tenets of the Declaration of Helsinki.

Consent to participate Informed consent was obtained from all individual participants included in the study.

Consent to publish Patients signed informed consent regarding publishing their data.

Disclaimer The funding organizations had no role in the design or conduct of this research. They provided unrestricted grants.

Open Access This article is licensed under a Creative Commons Attribution 4.0 International License, which permits use, sharing, adaptation, distribution and reproduction in any medium or format, as long as you give appropriate credit to the original author(s) and the source, provide a link to the Creative Commons licence, and indicate if changes were made. The images or other third party material in this article are included in the article's Creative Commons licence, unless indicated otherwise in a credit line to the material. If material is not included in the article's Creative Commons licence and your intended use is not permitted by statutory regulation or exceeds the permitted use, you will need to obtain permission directly from the copyright holder. To view a copy of this licence, visit http://creativecommons.org/licenses/by/4.0/.

\section{References}

1. Alio JL, Toffaha BT (2013) Refractive surgery with phakic intraocular lenses: an update. Int Ophthalmol Clin 53(1):91-110. https:// doi.org/10.1097/IIO.0b013e318272d27b

2. McAlinden C (2012) Corneal refractive surgery: past to present. Clin Exp Optom 95(4):386-398. https://doi.org/10.1111/j.14440938.2012.00761.x

3. Budo C, Hessloehl JC, Izak M, Luyten GP, Menezo JL, Sener BA, Tassignon MJ, Termote H, Worst JG (2000) Multicenter study of the Artisan phakic intraocular lens. J Cataract Refract Surg 26(8): 1163-1171

4. Huang D, Schallhorn SC, Sugar A, Farjo AA, Majmudar PA, Trattler WB, Tanzer DJ (2009) Phakic intraocular lens implantation for the correction of myopia: a report by the American Academy of Ophthalmology. Ophthalmology 116(11):2244-2258. https://doi. org/10.1016/j.ophtha.2009.08.018

5. Benedetti S, Casamenti V, Benedetti M (2007) Long-term endothelial changes in phakic eyes after Artisan intraocular lens implantation to correct myopia: five-year study. J Cataract Refract Surg 33(5):784-790. https://doi.org/10.1016/j.jcrs.2007.01.037

6. Perez-Santonja JJ, Iradier MT, Sanz-Iglesias L, Serrano JM, Zato MA (1996) Endothelial changes in phakic eyes with anterior chamber intraocular lenses to correct high myopia. J Cataract Refract Surg 22(8):1017-1022

7. Saxena R, Boekhoorn SS, Mulder PG, Noordzij B, van Rij G, Luyten GP (2008) Long-term follow-up of endothelial cell change after Artisan phakic intraocular lens implantation. Ophthalmology 115(4):608-613.e601. https://doi.org/10.1016/j.ophtha.2007.05. 036

8. Chebli S, Rabilloud M, Burillon C, Kocaba V (2018) Corneal endothelial tolerance after iris-fixated phakic intraocular lens implantation: a model to predict endothelial cell survival. Cornea 37(5): 591-595. https://doi.org/10.1097/ico.0000000000001527

9. Yuan X, Ping HZ, Hong WC, Yin D, Ting Z (2012) Five-year follow-up after anterior iris-fixated intraocular lens implantation in phakic eyes to correct high myopia. Eye (London, England) 26(2):321-326. https://doi.org/10.1038/eye.2011.292

10. Pop M, Mansour M, Payette Y (1999) Ultrasound biomicroscopy of the iris-claw phakic intraocular lens for high myopia. J Refract Surg (Thorofare, NJ : 1995) 15(6):632-635

11. Baikoff G (2006) Anterior segment OCT and phakic intraocular lenses: a perspective. J Cataract Refract Surg 32(11):1827-1835. https://doi.org/10.1016/j.jcrs.2006.08.025

12. Doors M, Berendschot TT, Hendrikse F, Webers CA, Nuijts RM (2009) Value of preoperative phakic intraocular lens simulation using optical coherence tomography. J Cataract Refract Surg 35(3):438-443. https://doi.org/10.1016/j.jcrs.2008.11.033

13. Doors M, Cals DW, Berendschot TT, de Brabander J, Hendrikse F, Webers CA, Nuijts RM (2008) Influence of anterior chamber morphometrics on endothelial cell changes after phakic intraocular lens implantation. J Cataract Refract Surg 34(12):2110-2118. https:// doi.org/10.1016/j.jcrs.2008.08.023

14. Baumeister M, Buhren J, Kohnen T (2002) Scheimpflug imaging of modern foldable high-refractive silicone and hydrophobic acrylic intraocular lenses. Dev Ophthalmol 34:187-194

15. Kohnen T, Cichocki M, Koss MJ (2008) Position of rigid and foldable iris-fixated myopic phakic intraocular lenses evaluated by Scheimpflug photography. J Cataract Refract Surg 34(1):114120. https://doi.org/10.1016/j.jcrs.2007.08.037 
16. Tehrani M, Schaefer M, Koeppe J, Dick HB (2007) Preoperative simulation of postoperative iris-fixated phakic intraocular lens position and simulation of aging using high-resolution Scheimpflug imaging. J Cataract Refract Surg 33(1):11-14. https://doi.org/10. 1016/j.jcrs.2006.07.039

17. Perez-Santonja JJ, Alio JL, Jimenez-Alfaro I, Zato MA (2000) Surgical correction of severe myopia with an angle-supported phakic intraocular lens. J Cataract Refract Surg 26(9):1288-1302

18. de Souza RF, Allemann N, Forseto A, Barros PS, Chamon W, Nose W (2003) Ultrasound biomicroscopy and Scheimpflug photography of angle-supported phakic intraocular lens for high myopia. J Cataract Refract Surg 29(6):1159-1166

19. Jonker SMR, Berendschot T, Ronden AE, Saelens IEY, Bauer NJC, Nuijts R (2018) Long-term endothelial cell loss in patients with artisan myopia and artisan toric phakic intraocular lenses: 5and 10-year results. Ophthalmology 125(4):486-494. https://doi. org/10.1016/j.ophtha.2017.08.011

20. McAlinden C, Khadka J, Pesudovs K (2011) Statistical methods for conducting agreement (comparison of clinical tests) and precision (repeatability or reproducibility) studies in optometry and ophthalmology. Ophthalmic Physiol Opt 31(4):330-338. https://doi.org/ 10.1111/j.1475-1313.2011.00851.x

21. Altman DG (1991) Practical Statistics for Medical Research. Chapman\% Hall Ltd, New York

22. Ferreira TB, Portelinha J (2014) Endothelial distance after phakic iris-fixated intraocular lens implantation: a new safety reference. Clin Ophthalmol (Auckland, NZ) 8:255-261. https://doi.org/10. 2147/opth.S56484

23. Doors M, Cruysberg LP, Berendschot TT, de Brabander J, Verbakel F, Webers CA, Nuijts RM (2009) Comparison of central corneal thickness and anterior chamber depth measurements using three imaging technologies in normal eyes and after phakic intraocular lens implantation. Graefe's archive for clinical and experimental ophthalmology $=$ Albrecht von Graefes Archiv fur klinische und experimentelle Ophthalmologie 247 (8):1139-1146. doi: https://doi.org/10.1007/s00417-009-1086-6

24. Kiraly L, Duncker G (2012) Biometry of the anterior eye segment for implantation of phakic anterior chamber lenses. A comparison of current measurement devices. Der Ophthalmologe : Zeitschrift der Deutschen Ophthalmologischen Gesellschaft 109(3):242-249. https://doi.org/10.1007/s00347-011-2520-9

25. Rosales P, Marcos S (2009) Pentacam Scheimpflug quantitative imaging of the crystalline lens and intraocular lens. J Refract Surg (Thorofare, NJ : 1995) 25(5):421-428

26. Fan R, Chan TC, Prakash G, Jhanji V (2018) Applications of corneal topography and tomography: a review. Clin Exp Ophthalmol 46(2):133-146. https://doi.org/10.1111/ceo.13136

27. Atchison DA, Charman WN (2011) Can partial coherence interferometry be used to determine retinal shape? Optom Vision Sci 88(5): E601-E607. https://doi.org/10.1097/OPX.0b013e318212ae56

28. Ang M, Baskaran M, Werkmeister RM, Chua J, Schmidl D, Aranha dos Santos V, Garhöfer G, Mehta JS, Schmetterer L (2018) Anterior segment optical coherence tomography. Prog Retin Eye Res 66:132-156. https://doi.org/10.1016/j.preteyeres.2018.04.002

Publisher's note Springer Nature remains neutral with regard to jurisdictional claims in published maps and institutional affiliations. 